\title{
Moduli spaces of Calabi-Yau $d$-folds as gravitational-chiral instantons
}

\section{Sergio Cecotti}

SISSA,

via Bonomea 265, I-34100 Trieste, Italy

E-mail: cecotti@sissa.it

ABstract: Motivated by the swampland program, we show that the Weil-Petersson geometry of the moduli space of a Calabi-Yau manifold of complex dimension $d \leq 4$ is a gravitational instanton (i.e. a finite-action solution of the Euclidean equations of motion of gravity with matter). More precisely, the moduli geometry of Calabi-Yau $d$-folds $(d \leq 4)$ describes instantons of (E)AdS Einstein gravity coupled to a standard chiral model.

From the point of view of the low-energy physics of string/M-theory compactified on the Calabi-Yau $X$, the various fields propagating on its moduli space are the couplings appearing in the effective Lagrangian $\mathscr{L}_{\text {eff }}$.

Keywords: Differential and Algebraic Geometry, Superstring Vacua, Classical Theories of Gravity, Sigma Models

ArXiv EPrint: 2007.09992 


\section{Contents}

1 Overview: moduli spaces as gravitational instantons 1

2 Details and proofs $\quad 5$

2.1 An informal sketch 5

2.2 Review of the $\Gamma \backslash G / K \sigma$-model $\quad 7$

$\begin{array}{lll}2.3 & \text { Pluri-harmonic maps } & 7\end{array}$

2.4 Review of $t t^{*}$ geometry 8

$\begin{array}{llr}2.5 & \text { Superconformal } t t^{*} \text { geometry } & 10\end{array}$

$\begin{array}{ll}2.6 & \text { Proof of eq. (1.9) } \\ & 11\end{array}$

$\begin{array}{lll}2.7 & \text { Review of }[24] & 11\end{array}$

2.8 Proof of eq. (1.10) 13

$\begin{array}{lll}2.9 & \text { Finite actions and finite volume } & 13\end{array}$

2.10 The canonical Kähler metrics for arbitrary $d \quad 14$

2.11 The explicit "Einstein equation" for arbitrary $d \quad 15$

Tis true without lying, certain $\&$ most true.

That wch is below is like that wch is above 8

that wch is above is like yt wch is below to do

ye miracles of one only thing.

The Emerald TABlet, translation by Isaac Newton

\section{Overview: moduli spaces as gravitational instantons}

The swampland program [1,2] (for reviews see [3, 4]) looks for a characterization of the effective field theories which arise as low-energy limits of consistent theories of quantum gravity, separating them from the vast swampland of effective theories which "look" consistent from a low-energy perspective, but cannot be completed to a fully consistent theory of quantum gravity. The program has produced a dozen or so conjectural necessary conditions (the "swampland conjectures" [1-4]) that all effective theories of quantum gravity should satisfy.

Dually, there is an inverse-swampland procedure. If we know that a certain effective theory does arise from quantum gravity, we may apply the swampland ideas to predict properties of the model. Often such properties are too fancy for anybody to have enough fantasy to guess them, and they escaped us when we looked at these theories with preswampland eyes. With post-swampland insight we know better. This short note illustrates a simple application of the inverse-swampland strategy. The result echos the opening quotation, which was a major inspiration for Newton in formulating his own consistent theory of gravity. 
We focus on the Weil-Petersson (WP) geometry of moduli spaces of compact CalabiYau (CY) manifolds $X_{d}$ of complex dimension $d \leq 4$. These CY manifolds describe stable supersymmetric vacua in string/M-theory, and the quantum-consistent low-energy effective theories around these vacua are captured by the geometry of their moduli spaces. Hence inverse-swampland may yield new insights on the geometry of CY moduli.

We claim ${ }^{1}$ that for $d \leq 4$ the CY moduli geometry yields a finite-action solution to the classical equations of motion of a moduli-space field theory of the form

$$
\int_{M} d^{2 m} x \sqrt{\operatorname{det} G}\left(-\frac{1}{2 \kappa^{2}} R+\frac{1}{2} G^{\alpha \beta} h(\phi)_{a b} \partial_{\alpha} \phi^{a} \partial_{\beta} \phi^{b}+\Lambda\right),
$$

where $M$ is the CY moduli space, $G_{\alpha \beta}$ its metric, and $R$ its scalar curvature. In $d=1$ the only CY spaces are the elliptic curves; their moduli space has real dimension 2 , so in this case the Einstein term in (1.1) is topological, while the cosmological constant $\Lambda$ vanishes - eq. (1.1) reduces for $d=1$ to the classical Polyakov action of a string moving in the appropriate target space (which is also 2-dimensional). When $d=2$ the modulispace Newton constant $\kappa^{2}$ is an adjustable parameter (this freedom reflects the fact that the moduli metric is always Einstein for CY 2-folds). For $d=3,4$ the Newton constant depends only on the dimension $d$ of the CY, while the cosmological constant $\Lambda$ depends on $d$ and the complex dimension $m$ of the moduli space:

$$
\frac{1}{\kappa^{2}}=\left\{\begin{array}{ll}
\text { free parameter } & d=2 \\
\operatorname{gcd}(d, 2) & d=3,4
\end{array}, \quad \Lambda=-(m-1)\left(\frac{m}{\kappa^{2}}+d\right) .\right.
$$

When $m=1$ the real dimension of the moduli space $M$ is 2 and $\Lambda=0$, and again (1.1) reduces to the classical Polyakov action. For $m>1$ the moduli-space gravity is "dynamical", and the cosmological constant negative. For $m=2$ we get instantons of a "realistic" four-dimensional gravity with matter. In the $d=2$ case the matter decouples in the limit $\kappa^{2} \rightarrow 0$, and the moduli-space Einstein equations reduce to $R_{\alpha \beta}=-m G_{\alpha \beta}$. In this limit it is clear that the finiteness of the moduli volume (one of the swampland conjectures [2-4]) should be really understood as a finite action condition for the moduli-space field theory (1.1). This observation applies in general.

The matter part of the action (1.1) is a standard $\sigma$-model with target a locally symmetric space $\Gamma \backslash G(\mathbb{R}) / K$, where the non-compact real Lie group $G \equiv G(\mathbb{R})$ is the automorphism group of the corresponding Griffiths period domain, that is, explicitly

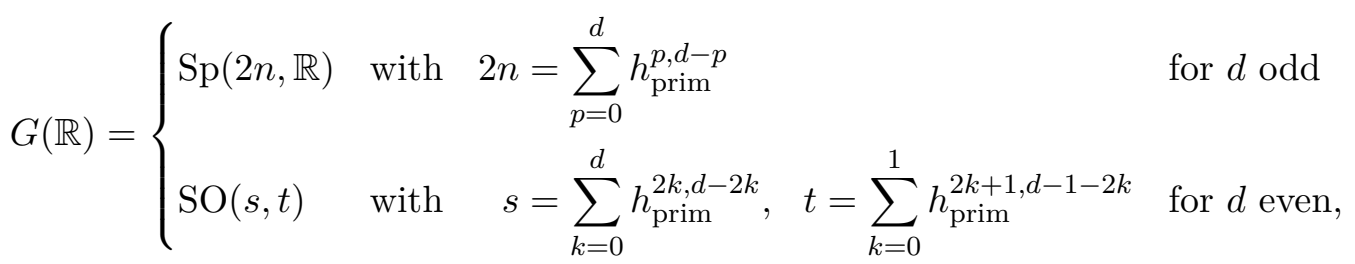

where $\left\{h_{\text {prim }}^{p, d-p}\right\}$ are the primitive Hodge numbers in middle dimension ${ }^{2}$

$$
h_{\text {prim }}^{p, d-p} \stackrel{\text { def }}{=} \operatorname{dim}_{\mathbb{C}}\left\{\xi \in \mathbb{H}^{p, d-p}\left(X_{d}\right): \omega \wedge \xi=0\right\} \quad \omega \equiv \text { Kähler form. }
$$

\footnotetext{
${ }^{1}$ The sharp version of the Claim will be presented momentarily, after fixing the necessary notation.

${ }^{2} \mathbb{H}^{p, q}\left(X_{d}\right)$ stands for the space of harmonic forms of type $(p, q)$ on $X_{d}$.
} 
$K \subset G(\mathbb{R})$ is a maximal compact subgroup. For comparison, we recall that the Griffiths period domain $D$ is the reductive coset $G(\mathbb{R}) / H$ where [5-7]

$$
H=\mathrm{SO}\left(h_{\text {prim }}^{d / 2, d / 2}\right) \times \prod_{0 \leq p<d / 2} \mathrm{U}\left(h_{\text {prim }}^{p, d-p}\right) \subset K .
$$

One may replace $G(\mathbb{R})$ with the Lie subgroup $M T(\mathbb{R}) \subseteq G(\mathbb{R})$ given by the real locus of the Mumford-Tate group $M T$ [8-10] of the moduli of $X_{d}$. Indeed the relevant matter field configuration $\phi: M \rightarrow \Gamma \backslash \Gamma(\mathbb{R}) / K$ has image in the totally geodesic submanifold ${ }^{3}$

$$
\Gamma \backslash M T(\mathbb{R}) /[M T(\mathbb{R}) \cap K] \hookrightarrow \Gamma \backslash G(\mathbb{R}) / K .
$$

The discrete group $\Gamma \subset M T(\mathbb{Z}) \subseteq G(\mathbb{Z})$ is the monodromy group of the CY period map.

Very roughly speaking, the moduli space has the form $M=\mathcal{G} \backslash \widetilde{M}$, with $\widetilde{M}$ diffeomorphic to $\mathbb{R}^{2 m}$ and $\mathcal{G}$ a discrete group with a neat subgroup of finite index. We call $\mathcal{G}$ the $U$-duality group. (In the present context it is isomorphic to the monodromy group $\Gamma$, but we denote them with distinct symbols for clarity).

A classical solution to (1.1) consists of two pieces of data: a metric $G_{\alpha \beta}$ on $\widetilde{M}$ admitting $\mathcal{G}$ as a group of isometries, and a harmonic map $\tilde{\phi}: \widetilde{M} \rightarrow G(\mathbb{R}) / K$ satisfying the $\mathcal{G}$ equivariant condition

$$
\tilde{\phi}(g \cdot x)=\rho(g) \cdot \tilde{\phi}(x), \quad x \in \widetilde{M}, \quad g \in \mathcal{G}
$$

for some group homomorphism

$$
\rho: \mathcal{G} \rightarrow \Gamma \equiv \rho(\mathcal{G}) \subset G(\mathbb{Z}) \subset G(\mathbb{R})
$$

called the monodromy representation. When (1.7) holds, one also says that the map $\tilde{\phi}$ is twisted by $\rho$. A $\rho$-twisted map descends to a map $\phi: M \rightarrow \Gamma \backslash G(\mathbb{R}) / K$, and we shall use $\tilde{\phi}$ and $\phi$ interchangeably. Our claim states that the CY moduli geometry is given by a pair $\left(G_{\alpha \beta}, \tilde{\phi}\right)$ which satisfies the equations of motion following from the action (1.1):

$$
\begin{aligned}
& D^{\alpha} \partial_{\alpha} \tilde{\phi}=0 \\
& R_{\alpha \beta}-\frac{1}{2} G_{\alpha \beta} R+\kappa^{2} \Lambda G_{\alpha \beta}=\kappa^{2} T_{\alpha \beta},
\end{aligned}
$$

where the derivative $D_{\alpha}$ is covariant for the combined Levi-Civita connections of $T^{*} M$ and $\tilde{\phi}^{*} T(G / K)$. Eq. (1.9) just expresses the fact that $\tilde{\phi}$ is a harmonic map $\widetilde{M} \rightarrow G(\mathbb{R}) / K$ for the source-space metric $G_{\alpha \beta}$.

Having fixed the notation, let us make our claim sharp:

Claim. Let $G_{\alpha \beta}$ be the Weil-Petersson metric on the complex moduli space $M$ of a $C Y$ $d$-fold, with $d \leq 4$, and let $\phi$ be the composed map $\phi \equiv \pi \circ p$ where

$$
\begin{array}{ll}
p: M \rightarrow \Gamma \backslash G(\mathbb{R}) / H & \text { the (global) Griffiths period map [5-10] } \\
\pi: \Gamma \backslash G(\mathbb{R}) / H \rightarrow \Gamma \backslash G(\mathbb{R}) / K & \text { the canonical projection. }
\end{array}
$$

Then the pair $\left(G_{\alpha \beta}, \phi\right)$ is a finite-action solution to eqs. (1.9), (1.10) with constants as in eq. (1.2).

\footnotetext{
${ }^{3}$ This statement follows from the structure theorem for the period map [8-10].
} 
Remark 1. Eq. (1.9) remains true when $d \geq 5$. The WP metric $G_{\alpha \beta}$ still satisfies an "Einstein-like" equation. However it seems that one cannot construct an off-shell action with positive kinetic terms whose canonical energy-momentum tensor yields the source term in the equation. This is to be expected since the moduli geometry of $d \geq 5$ Calabi-Yau's is not required to have "magical" properties by swampland consistency conditions.

The moduli space fields $\left(G_{\alpha \beta}, \phi\right)$ as effective couplings. To make explicit contact with the swampland program, let us recall the low-energy $4 \mathrm{~d}$ effective Lagrangian of Type IIB compactified on the (simply-connected) CY 3-fold $X_{3}$

$$
\mathscr{L}_{\mathrm{IIB}}=\sqrt{-g}\left(-\frac{1}{2} R+\frac{1}{2} G(\varphi)_{\alpha \beta} \partial^{\mu} \varphi^{\alpha} \partial_{\mu} \varphi^{\beta}+\frac{i}{16 \pi} \tau(\varphi)_{a b} F_{+}^{a} F_{+}^{b}-\frac{i}{16 \pi} \bar{\tau}(\varphi)_{a b} F_{-}^{a} F_{-}^{b}+\cdots\right),
$$

where for brevity we wrote only the matter terms involving the bosonic fields of the vectormultiplets. The metric $G_{\alpha \beta}$ appearing in the vector-multiplet scalars' kinetic terms coincides with the Weil-Petersson metric on the moduli space $M$ of $X_{3}$ [11, 12]. For a fixed point $\varphi \in \widetilde{M}$, the gauge coupling $\tau(\varphi)_{a b}$ is a symmetric complex matrix with positive imaginary part, that is, a point in the Siegel upper half-space

$$
\left.\tau(\varphi)_{a b} \in \mathrm{Sp}\left(2 h^{2,1}+2, \mathbb{R}\right) / \mathrm{U}\left(h^{2,1}+1\right) \equiv G(\mathbb{R}) / K \quad \text { (cf. eq. (1.3) with } d=3\right) .
$$

Hence the $4 \mathrm{~d}$ gauge coupling may be identified with the map

$$
\tilde{\phi}: \widetilde{M} \rightarrow G(\mathbb{R}) / K, \quad \tilde{\phi}: \varphi \mapsto \tau(\varphi)_{a b} .
$$

However this way of describing the gauge couplings is not intrinsic, since $\tau(\varphi)_{a b}$ depends on a choice of duality frame. Even worse: the $\tau(\varphi)_{a b}$ are multi-valued ${ }^{4}$ functions on $M$ because when we go around a non-trivial loop in $M$ we come back with a rotated electromagnetic duality frame. The intrinsic description of the gauge couplings is instead given by the quotient map

$$
\phi: M \equiv \mathcal{G} \backslash \widetilde{M} \rightarrow \Gamma \backslash G(\mathbb{R}) / K, \quad \phi:[\varphi] \mapsto\left[\tau(\varphi)_{a b}\right] .
$$

In other words, the lifted gauge coupling map $\tilde{\phi}$ is twisted by the monodromy representation $\rho$ as in eqs. (1.7), (1.8). Indeed, the $U$-duality group $\mathcal{G}$ acts both on $\widetilde{M}$ (by isometries) and on the vector field-strengths (by electro-magnetic dualities) while leaving the physical energy-momentum tensor $T_{\mu \nu}$ invariant; this entails that the 'naive' gauge coupling map $\tilde{\phi}$ is twisted by the monodromy representation $\rho$ of $\mathcal{G}$. This being understood, the on-shell configurations (1.11), (1.12) of the two fields $\left(G_{\alpha \beta}, \phi\right)$ which propagate in the moduli space $M$ are exactly the same as the couplings appearing in the Type IIB $4 \mathrm{~d}$ effective Lagrangian $\mathscr{L}_{\text {IIB }}$ :

$$
\begin{aligned}
G_{\alpha \beta} & \equiv \text { vector-multiplet scalars' metric } \\
\phi & \equiv \text { (intrinsically-defined) gauge couplings, eq. (1.16). }
\end{aligned}
$$

\footnotetext{
${ }^{4}$ The structure theorem yields the dichotomy: either the gauge couplings $\tau(\varphi)_{a b}$ are field-independent numerical constants (as in the case of rigid CY 3-folds [13]) or $\tau(\varphi)_{a b}$ must be multivalued.
} 
Remark 2. More generally, it is pretty obvious that all couplings appearing in the Lagrangian $\mathscr{L}_{\text {eff }}$ of any $4 \mathrm{~d}$ supergravity which is consistent with the swampland conjectures [1-4] and has $\geq 8$ supercharges describe (as functions of the scalar fields) gravitational instantons. Again, the finite volume conjecture gets re-interpreted as the statement that the field configuration in moduli space which decribes the effective couplings in $\mathscr{L}_{\text {eff }}$ has finite action.

Remark 3. Conversely, the $\mathcal{N} \geq 2$ SUGRA's which do not satisfy the swampland conjectures are not gravitational instantons. E.g. the homogeneous $\mathcal{N}=2$ models constructed in [14], all of which fall in the swampland [15], do satisfy eqs. (1.9), (1.10) but have infinite action.

\section{Details and proofs}

Of course, once we have strong reasons - such as the swampland story - to believe that something ought to be true, we look for actual proofs rather than relying on widely believed conjectures. Our treatment in this note will be totally rigorous (except that we do not discuss the singularities of the relevant solution - a crucial issue, but not one consistent with the purpose of writing a short note).

We present an informal discussion of the general picture in section 2.1. Then in sections 2.2-2.11 we enter in the technical details, and write explicit expressions for all relevant quantities.

\subsection{An informal sketch}

There are two approaches (or languages) for the geometry of Calabi-Yau moduli spaces: (i) Griffiths theory of variations of Hodge structures (VHS) [5-7], and (ii) $t t^{*}$ geometry [16-18]. Equivalence of the two viewpoints (in the appropriate contexts) was proven in $[16,19]$ (and enshrined in the math literature as a theorem in [20]). We shall use both languages, with a preference for the second one.

On the moduli space of a Calabi-Yau $d$-fold there is an infinite family of a priori distinct canonical Kähler metrics. From the point of view of VHS this plethora arises because the Griffiths period domain $D \equiv G(\mathbb{R}) / H[5-7]$ carries several holomorphic homogeneous line bundles whose canonical connection has a curvature which is positive when restricted to the Griffiths horizontal tangent bundle. The pull-back to $M$, via the period map $p: M \rightarrow \Gamma \backslash D$, of any one of these curvatures yields a Kähler form on $M$. There is one horizontally-positive line-bundle which exists on the period domain $D$ for all Hodge numbers $\left\{h^{p, q}\right\}$, namely the Griffiths canonical line bundle [21]. The corresponding Kähler metric is called the Hodge metric $K_{j \bar{k}}$, and is the best behaved one in the family. In the case of Calabi-Yau $d$-folds, one has $h^{d, 0}=1$ and there is another important horizontally-positive line bundle whose sections are the holomorphic $(d, 0)$-forms. Its curvature defines the Weil-Petersson (WP) Kähler metric $G_{j \bar{k}} . \quad K_{j \bar{k}}, G_{j \bar{k}}$ do not exhaust the list of canonical VHS metrics. Taking linear combinations with positive coefficients of the several canonical metrics, we construct 
a convex cone $\mathscr{C}_{d}$ of God-given Kähler metrics on the moduli $M$. The term "God-given" here has a precise technical meaning:

the $U$-duality group $\mathcal{G}$ acts by isometries with respect to

all Kähler metrics in the convex cone $\mathscr{C}_{d}$.

This is quite remarkable, since $\mathcal{G}$ is a "huge" group: for CY 3-folds, say, unless the IIB $4 \mathrm{~d}$ gauge couplings $\tau(\varphi)_{a b}$ are numerical constants (i.e. the CY is rigid [13]), the Zariski closure of $\mathcal{G}$ is a semi-simple real Lie group of positive dimension [15]. We stress that $\mathscr{C}_{d}$ is a cone of actual Kähler metrics, not just Kähler classes. We write $K_{j \bar{k}}^{(c)}$ for the Kähler metric associated to a point $c \in \mathscr{C}_{q}$.

Not all the canonical Kähler metrics are independent: decreasing the dimension $d$ of the Calabi-Yau space, the dimension of the cone gets smaller and smaller

$$
\begin{aligned}
\operatorname{dim} \mathscr{C}_{d} & =\operatorname{rank}(\text { group of homogeneous line-bundles over } G / H) \equiv \\
& \equiv \operatorname{rank} \operatorname{Hom}(H, \mathrm{U}(1)) \leq\left[\frac{d+1}{2}\right] \quad \begin{array}{l}
\text { equality for connected } \\
\text { Hodge structures }
\end{array}
\end{aligned}
$$

For $d \leq 4$ the inequality is saturated provided the CY is not rigid.

The Ricci tensor $R_{j \bar{k}}$ of the WP metric $G_{j \bar{k}}$ is also the pull-back of the curvature of a homogenous line-bundle on $D$, hence $R_{j \bar{k}}$ belongs to the linear span of the God-given metrics, i.e. $R_{j \bar{k}}$ can be written as a linear combination of canonical VHS metrics. This is consistent since all Kähler metrics $K_{j \bar{k}}^{(c)}$ satisfy the same Bianchi identity as the WP Ricci tensor

$$
D^{j}\left(K_{j \bar{k}}^{(c)}-\frac{1}{2} G_{j \bar{k}} K^{(c)}\right)=0, \quad \text { where } K^{(c)} \stackrel{\text { def }}{=} 2 G^{j \bar{k}} K_{j \bar{k}}^{(c)},
$$

and $D_{j}$ is the Levi-Civita connection of the WP metric. On the moduli space of CY $d$-folds we have an identity of the form

$$
R_{j \bar{k}}-\frac{1}{2} G_{j \bar{k}} R=\sum_{c=1}^{\operatorname{dim} \mathscr{C}_{d}} \lambda^{(c)}\left(K_{j \bar{k}}^{(c)}-\frac{1}{2} G_{j \bar{k}} K^{(c)}\right)
$$

for certain numerical constants $\lambda^{(c)}$ (to be computed in section 2.11 below). We conclude that the WP metric $G_{j \bar{k}}$ is a solution to the Einstein equations provided the r.h.s. of (2.3) may be written as a physically sound energy-momentum tensor plus a cosmological constant term.

For CY's of dimension $d=1,2$ one has $\operatorname{dim} \mathscr{C}_{d}=1$, so all canonical VHS metrics are multiples of the WP one which then must be Einstein, i.e. $R_{j \bar{k}}=-\Lambda G_{j \bar{k}}$ for some $\Lambda$.

For $d=3,4$ there is a 2-parameter family of VHS Kähler metrics on $M$, and then the Ricci tensor of the WP metric must be a linear combination of the WP metric $G_{j \bar{k}}$ and the Hodge one $K_{j \bar{k}}$. Now our Claim follows from the fact ${ }^{5}$ that the combination entering in $(2.3)$

$$
K_{j \bar{k}}-\frac{1}{2} G_{j \bar{k}} K \equiv T_{j \bar{k}},
$$

is nothing else than the canonical energy-momentum tensor of the $\Gamma \backslash G(\mathbb{R}) / K \sigma$-model evaluated on the on-shell field configuration (1.11), (1.12).

Having sketched the general picture, let us now write the explicit formulae.

\footnotetext{
${ }^{5}$ See eq. (2.31) below.
} 


\subsection{Review of the $\Gamma \backslash G / K \sigma$-model}

$G \equiv G(\mathbb{R})$ is a non-compact real Lie group and $K \subset G$ a maximal compact subgroup which is fixed by the Cartan involution $\theta$. A map $M \rightarrow \Gamma \backslash G / K$ can be lifted (non-uniquely) to a map $E: \widetilde{M} \rightarrow G$. We see the lifted map as a field $E(x)$ on the Euclidean space-time $\widetilde{M}$ which takes value in the concrete matrix group given by the Hodge representation of $G(\mathbb{R})$ which, for the groups in (1.3), is the fundamental one $(\mathbf{2} \boldsymbol{n}$ and, respectively, $\boldsymbol{s}+\boldsymbol{t})$. The field $E(x)$ is twisted by the monodromy representation (cf. eq. (1.7))

$$
E(g \cdot x)=\rho(g) \cdot E(x), \quad \forall g \in \mathcal{G},
$$

so it descends to a field (or map) $\underline{E}: M \rightarrow \Gamma \backslash G$. Two field configurations, $E(x)$ and $E(x)^{\prime}$, which differ by the multiplication on the right by a position-dependent element of $K$, are declared to be gauge-equivalent (i.e. the same physical configuration)

$$
E(x)^{\prime} \sim E(x) \quad \Longleftrightarrow \quad E(x)^{\prime}=E(x) U(x), \quad \text { with } U(x) \in K .
$$

By a global field configuration $E(x)$ we actually mean a local lift $E(x)_{\alpha}: U_{\alpha} \rightarrow G$ for each open set of a cover $\cup_{\alpha} U_{\alpha}=\widetilde{M}$, with transition function $E(x)_{\beta}^{-1} E(x)_{\alpha} \in K$ in $U_{\alpha} \cap U_{\beta}$.

We have the $K$-principal bundle $\varpi: G \rightarrow G / K$, and the physical gauge-invariant map is $\varpi \circ E: \widetilde{M} \rightarrow G / K$ or, more precisely, its $\mathcal{G}$-equivariant quotient

$$
\underline{\varpi} \circ \underline{E}: M \rightarrow \Gamma \backslash G / K \text {. }
$$

We adopt the following notation: for $a \in \mathfrak{g}$ (the Lie algebra of $G$ ), $a^{\mathrm{e}}$ and $a^{\mathrm{o}}$ denote, respectively, the projection on the even and odd parts under the Cartan involution $\theta$. The action of the $\sigma$-model with target space $\Gamma \backslash G / K$ is

$$
\frac{1}{2} \int_{M} d^{2 m} x \sqrt{\operatorname{det} G} G^{\alpha \beta} \operatorname{tr}\left[\left(E^{-1} \partial_{\alpha} E\right)^{\mathrm{o}}\left(E^{-1} \partial_{\beta} E\right)^{\mathrm{o}}\right]
$$

(one checks that it is $K$ gauge invariant). The energy-momentum tensor is

$$
T_{\alpha \beta}=\operatorname{tr}\left[\left(E^{-1} \partial_{\alpha} E\right)^{\mathrm{o}}\left(E^{-1} \partial_{\beta} E\right)^{\mathrm{o}}\right]-\frac{1}{2} G_{\alpha \beta} G^{\gamma \delta} \operatorname{tr}\left[\left(E^{-1} \partial_{\gamma} E\right)^{\mathrm{o}}\left(E^{-1} \partial_{\delta} E\right)^{\mathrm{o}}\right] .
$$

The equations of motion say (by definition) that the field $E$ is on-shell if and only if the corresponding physical map, $\underline{\varpi} \circ \underline{E}: M \rightarrow \Gamma \backslash G / K$, is harmonic.

\subsection{Pluri-harmonic maps}

Let $M$ be a Kähler manifold and $Y$ any Riemannian manifold. A map $f: M \rightarrow Y$ is pluri-harmonic iff

$$
D_{j} \partial_{\bar{k}} f=0 .
$$

Note that the covariant derivative $D_{j}$ contains only the Levi-Civita connection of $f^{*} T Y$. If $f$ is pluri-harmonic, $G^{j \bar{k}} D_{j} \partial_{\bar{k}} f \equiv 0$, so $f$ is a fortiori harmonic, hence a classical solution of the $\sigma$-model with target space $Y$ and source space $M$. We stress that (2.10) does not contain the Kähler metric of $M$, so a pluri-harmonic map is harmonic for all choices of Kähler metric. 
In our application $Y$ is the locally symmetric space $\Gamma \backslash G / K$ which is non-compact of finite volume. ${ }^{6}$ We assume $M$ to be non-compact and the existence of some complete Kähler metric $\stackrel{\circ}{g}_{j \bar{k}}$ on $M$ of finite volume; "some" means that the reference metric $\stackrel{\circ}{g}_{j \bar{k}}$ may have nothing to do with the physical metric $G_{j \bar{k}} \cdot{ }^{7}$ We claim that in these circumstances ${ }^{8}$ any classical solution $E_{0}$ of the $\sigma$-model (defined with the space-time metric $\stackrel{\circ}{g}_{j \bar{k}}$ ) which has finite action

$$
S\left(E_{0}\right) \equiv \int_{M} d^{2 m} x(\operatorname{det} \stackrel{\circ}{g}) \stackrel{\circ}{g} j \bar{k} \operatorname{tr}\left[\left(E_{0}^{-1} \partial_{j} E_{0}\right)^{\circ}\left(E_{0}^{-1} \partial_{\bar{k}} E_{0}\right)^{\circ}\right]<\infty
$$

is automatically pluri-harmonic, hence a solution of the equations of motion for any other choice of Kähler metric $G_{j \bar{k}} \neq \stackrel{\circ}{g}_{j \bar{k}}$ on the source space $M$. The claim is a special case of a more deep fact, crucial for the swampland story, which shall be discussed elsewhere in its proper context. A sketch of the proof (for the special situation at hand) will be given in the next paragraph after the introduction of the necessary notation.

\subsection{Review of $t t^{*}$ geometry}

A $t t^{*}$ geometry on the complex manifold $M$ is just a pluri-harmonic map $M \rightarrow \Gamma \backslash G / K$ of finite action, ${ }^{9}$ where $G, K$ and $\Gamma$ are as in section 2.2. As in that paragraph, the $t t^{*}$ map may be lifted to a map $S: \widetilde{M} \rightarrow G$. Again, we see $S$ as a field on $\widetilde{M}$ taking value in the concrete matrix group $G$. In facts, $S$ is just a special instance of the $\sigma$-model field $E$ of section 2.2: $S$ is not just an on-shell field configuration, it satisfies the stronger condition of being pluriharmonic (this is essentially automatic in the present circumstances, see below). $S$ has a direct physical meaning: in the $t t^{*}$ literature $[18,25,26] S$ is called the BPS brane amplitude (for some value $\zeta=e^{i \theta}$ of the spectral parameter which depends on the chosen lift).

Since $M$ is complex, we may decompose the differential forms into definite type

$$
\left(S^{-1} d S\right)^{\mathrm{e}}=A+\bar{A}, \quad\left(S^{-1} d S\right)^{\mathrm{o}}=C+\bar{C},
$$

where unbarred (barred) stands for type $(1,0)$ (resp. $(0,1))$. We introduce the $K$-covariant Dolbeault differentials $D=\partial+A$ and $\bar{D}=\bar{\partial}+\bar{A}$. We have the identity

$$
D C=\left.\left(\left(d+S^{-1} d S\right)^{2}\right)^{\mathrm{o}}\right|_{(2,0)-\text { part }}=0
$$

while the condition that the $\mathcal{G}$-twisted $t t^{*}$ map $\varpi \circ S: \widetilde{M} \rightarrow G / K$ is pluri-harmonic reads

$$
\bar{D} C=0 .
$$

\footnotetext{
${ }^{6}$ More precisely: we may reduce to the case of finite volume without loss of generality. If $\Gamma$ is thin, replace it by an arithmetic group which contains it.

${ }^{7}$ Actually, taking as reference metric $\stackrel{\circ}{g}_{j \bar{k}}$ the physical one $G_{j \bar{k}}$ only improves the situation.

${ }^{8}$ For the statement to be true, one needs to require some extra "regularity" conditions which are tautologically satisfied for the spaces of interest.

${ }^{9}$ In this note we add to the "standard" definition of $t t^{*}$ geometry the condition that the underlying pluri-harmonic map has finite action; all $t t^{*}$ geometries arising from physics satisfy this condition. For the geometries relevant for the present paper this will be shown in section 2.6 below.
} 
Before proceeding, let us pause a while to sketch the idea behind the claim made at the end of the previous paragraph: if $M$ is a non-compact Kähler manifold of finite volume, satisfying some mild regularity condition, then a harmonic map $M \rightarrow \Gamma \backslash G / K$ of finite-energy is automatically pluri-harmonic. One starts from Simpson's Bochner-formula [20, 27] for harmonic maps with source space a Kähler manifold $M$. In the present set-up and notations, this Bochner-formula takes the form (see eqs. (4.1)-(4.8) of [15])

$$
\varpi \circ S \text { harmonic } \Rightarrow \bar{D}^{i} \bar{D}^{j} \operatorname{tr}\left(C_{i} C_{j}\right)=\|\bar{D} C\|^{2}+\text { non-negative. }
$$

When $M$ is compact, the integral of the total derivative in the l.h.s. vanishes, so the two non-negative terms in the r.h.s. should vanish separately, and we have $\bar{D} C=0$, which is the statement that the map $\varpi \circ S$ is pluri-harmonic. If $M$ is non-compact, the integral of the 1.h.s. yields a boundary term at infinity, and the same conclusion applies provided we can show that this boundary term vanishes. This vanishing condition at infinity holds when $M$ has finite volume and satisfies the mild technical assumptions (roughly: "the ends of $M$ at $\infty$ have vanishing size") while the harmonic map has finite-energy (i.e. it is "trivial at $\infty$ ").

We return to $t t^{*}$ geometry. A short computation $[15,28]$ shows that the compatibility condition of $(2.13)$ with $(2.14),[D, \bar{D}] C=0$, implies

$$
C \wedge C=0,
$$

which expresses the fact that the $t t^{*}$ chiral ring $\mathscr{R}$ is commutative. Using (2.13), (2.14), and (2.16) one checks that the Maurier-Cartan identity $\left(d+S^{-1} d S\right)^{2}=0$ is equivalent to the statement that the ${ }^{10} \mathfrak{g}^{\mathbb{C}}$-valued connection

$$
\nabla^{(\zeta)} \stackrel{\text { def }}{=} d+A+\bar{A}+\zeta C+\zeta^{-1} \bar{C}
$$

is flat for all values of the spectral parameter $\zeta \in \mathbb{P}^{1}$

$$
\left(\nabla^{(\zeta)}\right)^{2} \equiv 0
$$

Eq. (2.18) is the Lax form of the $t t^{*}$ PDEs $[16-18,28]$.

The application of $t t^{*}$ geometry to $2 \mathrm{~d}(2,2)$ QFT [16] works as follows. Let $P$ be the complex space of $F$-term ${ }^{11}$ parameters. Over $P$ we have the vector bundle $\mathscr{V}$ whose fiber at $p \in P$ is the space of SUSY vacua of the QFT with couplings $p$. The $t t^{*}$ connection $D+\bar{D}$ acts on $\mathscr{V}$; by eq. (2.18) it endows $\mathscr{V}$ with a holomorphic structure. By construction the $t t^{*}$ connection is metric for the QFT Hilbert space inner product, and hence it is the unique Chern connection on $\mathscr{V}$ (and also the Berry one). In a holomorphic gauge we have

$$
A=g \partial g^{-1}, \quad \bar{A}=0,
$$

where $g \equiv\left(g_{a \bar{b}}\right)$ is the Hilbert space Hermitian metric along the fibers (the $t t^{*}$ metric [16]). The spectral flow isomorphism [22] states that

$$
\mathscr{V} \cong \mathscr{R} \hookrightarrow \operatorname{End}(\mathscr{V}) \cong \mathscr{V} \otimes \mathscr{V}^{\vee} \cong \mathscr{V}^{\otimes 2},
$$

\footnotetext{
${ }^{10}$ Here and below $\mathfrak{g}^{\mathbb{C}} \equiv \mathfrak{g} \otimes \mathbb{C}$, where $\mathfrak{g}$ is the Lie algebra of the real Lie group $G$.

${ }^{11}$ The distinction between $F$-term and twisted $F$-term is a matter of convention. We loosely say " $F$-term" to mean either one, depending on the particular application one has in mind.
} 
where $\mathscr{R}$ is the holomorphic bundle whose fiber $\mathscr{R}_{p}$ is the chiral ring at $p \in P$. The last isomorphism in (2.20) is the reality structure ${ }^{12}[16]$. The holomorphic vacuum bundle $\mathscr{V}$ is then isomorphic to a sub-bundle of its tensor-square $\mathscr{V} \otimes 2$. This yields an induction on the bundle metrics: start with the fiber metric $g$ for $\mathscr{V}$; it induces a fiber metric for $\mathscr{V} \otimes 2$, and its restriction to the sub-bundle $\mathscr{R}$ is then a second fiber metric $h$ for $\mathscr{V}$ (one may iterate the process ad infinitum).

\subsection{Superconformal $t t^{*}$ geometry}

The discussion in section 2.4 applies to all 2d (2,2) QFTs [16]. When the (2,2) QFT is superconformal one is mainly interested in the $t t^{*}$ geometry restricted to the conformal submanifold $M \subset P$ of (exactly) marginal deformations. When so restricted, the holomorphic bundles $\mathscr{R} \rightarrow M$ and $\mathscr{V} \rightarrow M$ get graded by the superconformal U(1) charge $q$

$$
\mathscr{R}=\bigoplus_{q=0}^{\hat{c}} \mathscr{R}_{q}, \quad \mathscr{V}=\bigoplus_{q=-\hat{c} / 2}^{\hat{c} / 2} \mathscr{V}_{q}, \quad \mathscr{V}_{q}^{\vee} \cong \mathscr{V}_{-q}, \quad \mathscr{V}_{q-\hat{c} / 2} \cong \mathscr{R}_{q} .
$$

The decomposition of $\mathscr{V}$ is orthogonal for the $t t^{*}$ fiber metric $g$ [16]. Conformal perturbation theory gives us the isomorphism ${ }^{13}$

$$
\begin{aligned}
& \text { holomorphic tangent bundle } \\
& \text { of conformal manifold } M
\end{aligned} \quad T M \cong \mathscr{V}_{1-\hat{c} / 2} \cong \mathscr{R}_{1} \hookrightarrow \mathscr{V}^{\otimes 2} .
$$

The Hodge metric is the metric on $T M$ given by the induced metric on $\mathscr{R}_{1}$ as a sub-bundle of $\mathscr{V} \otimes 2$, while the WP metric is the normalized $t t^{*}$ metric restricted to $\mathscr{V}_{1-\hat{c} / 2}[16,24]$ :

$$
\text { WP metric on } M=\frac{\left.g\right|_{\mathscr{V}_{1-\hat{c} / 2}}}{g_{-\hat{c} / 2}}, \quad \text { Hodge metric on } M=\left.h\right|_{\mathscr{H}_{1-\hat{c} / 2}} \text {. }
$$

When the $t t^{*}$ geometry describes the complex moduli of a CY $d$-fold $X_{d}$ - that is, when the $2 \mathrm{~d}(2,2)$ SCFT is the $X_{d} \sigma$-model - one has $\hat{c}=d$ and

$$
\operatorname{rank} \mathscr{V}_{q}=h_{\text {prim }}^{d / 2-q / 2+q}, \quad \text { in particular, } \operatorname{rank} \mathscr{V}_{\mp \hat{c} / 2}=1 .
$$

In this case the $t t^{*}$ Lie group $G \equiv G(\mathbb{R})$, introduced in section 2.4 , is $\operatorname{Sp}(2 n, \mathbb{R})$ or $\operatorname{SO}(s, t)$ for $\hat{c}$ odd, respectively, even; that is, the $t t^{*}$ group $G(\mathbb{R})$ coincides with the VHS automorphism group (cf. eq. (1.3)). Moreover there is a $\mathrm{U}(1)$ grading element $Q \in \mathfrak{g} \otimes \mathbb{C}$ such that $^{14}[24]$

$$
[Q, C]=-C,\left.\quad Q\right|_{\mathscr{V}_{q}}=q \operatorname{Id}_{\mathscr{V}_{q}} .
$$

Refs. $[16,19,20]$ show that the VHS geometry of the complex moduli of a CY $d$-fold is described by a $t t^{*}$ geometry which satisfies the additional conditions $(2.21)-(2.25)$. The Lie sub-group $H \subset G$ (cf. eq. (1.5)) is the centralizer of the $\mathrm{U}(1)$ charge operator $Q$ in $G .{ }^{15}$

\footnotetext{
${ }^{12}$ Equivalently, the topological metric $\eta[23]$.

${ }^{13}$ In the VHS language this isomorphism is called the "local Torelli theorem".

${ }^{14}$ The adjoint action of $Q$ on $\mathfrak{g}$ gets transported on the bundles $\mathscr{V}_{q} \rightarrow M$ because these bundles are the pull-back (via the period map) of homogeneous bundles on the Griffiths domain. See, e.g. chapter 11 of [29].

${ }^{15}$ Here it is crucial that in the SCFT case the real Lie group $G$ is of "Mumford-Tate type" i.e. that it contains a compact maximal torus, i.e. $\operatorname{rank} G=\operatorname{rank} K[15]$.
} 


\subsection{Proof of eq. (1.9)}

The crucial fact is that a solution to $t t^{*}$ corresponds to a pluri-harmonic map

$$
\underline{\varpi} \circ \underline{E}: M \rightarrow \Gamma \backslash G / K,
$$

hence, in particular, to a solution of the $\sigma$-model (2.8). Since $\underline{\varpi} \circ \underline{E}$ is a solution for all Kähler metrics on $M$ (cf. section 2.3), the $t t^{*}$ map $\underline{\varpi} \circ \underline{E}$ is in particular harmonic for the WP metric $G_{j \bar{k}}$.

The particular $\sigma$-model solution which describes the moduli geometry of a CY moreover has finite action in the sense that

$$
\int_{M} d^{2 m} \sqrt{G} L_{\sigma \text {-model }}<\infty \quad \text { where } L_{\sigma \text {-model }} \equiv \frac{1}{2} G^{\alpha \beta} h(\phi)_{a b} \partial_{\alpha} \phi^{a} \partial_{\beta} \phi^{b}
$$

We defer the proof of (2.27) to section 2.9 below.

This shows eq. (1.9). The argument works for all dimensions $d$ of the Calabi-Yau.

\subsection{Review of $[24]$}

The Hodge metric $K_{j \bar{k}}$ was introduced in $t t^{*}$ geometry in ref. [24], and further studied in [30], for its relation with the $\tau$-function of isomonodromic problems and, respectively, the Ray-Singer torsion. As already mentioned, in VHS theory the Hodge metric makes sense in the complex moduli space of any projective variety, Calabi-Yau or not. Correspondingly, from a $t t^{*}$ perspective the Hodge metric should be a good Kähler metric for all $2 \mathrm{~d}(2,2)$ QFTs whether they are superconformal or not. When the $2 \mathrm{~d}$ theory is superconformal, however, the metric $K_{j \bar{k}}$ (restricted to the exactly marginal deformations) has nicer properties.

For a general $(2,2)$ QFT the Hodge metric reads [24]

$$
K_{j \bar{k}}=\operatorname{tr}\left[C_{j} \bar{C}_{\bar{k}}\right]
$$

where $C_{j}$ and $\bar{C}_{\bar{k}}$ are the coefficients of the matrix-valued 1-forms $C \equiv C_{j} d t^{j}$ and $\bar{C} \equiv \bar{C}_{\bar{k}} d \bar{t}^{\bar{k}}$ (cf. (2.12)); $\left\{t^{j}\right\}$ are complex coordinates in the parameter space $P$ of the (2,2) QFT [16].

In the superconformal case we restrict the 1 -forms $C, \bar{C}$ to the conformal submanifold $M \hookrightarrow P$, i.e. to marginal deformations. Conservation of the conformal U(1) charge yields

$$
\operatorname{tr}\left[C_{i} C_{j}\right]=0,
$$

and we can rewrite equation (2.28) in arbitrary real (that is, not necessarily holomorphic) local coordinates $x^{\alpha}$ in the form

$$
\begin{aligned}
d s_{\mathrm{HODGE}}^{2} \equiv K_{\alpha \beta} d x^{\alpha} d x^{\beta} & =\operatorname{tr}\left[(C+\bar{C})_{\alpha}(C+\bar{C})_{\beta}\right] d x^{\alpha} d x^{\beta}= \\
& =\operatorname{tr}\left[\left(S^{-1} \partial_{\alpha} S\right)^{\mathrm{o}}\left(S^{-1} \partial_{\beta} S\right)^{\mathrm{o}}\right] d x^{\alpha} d x^{\beta},
\end{aligned}
$$

where, in the second line, we used eq. (2.12). From eq. (2.9) we see that the energymomentum tensor of the $\sigma$-model, evaluated on the particular $t t^{*}$ on-shell field configuration $E=S$, is

$$
T_{\alpha \beta}=K_{\alpha \beta}-\frac{1}{2} G_{\alpha \beta} G^{\gamma \delta} K_{\gamma \delta} .
$$


In refs. $[24,30]$ there is a second formula for the Hodge metric - this one valid only along the conformal manifold $M$ of a superconformal $t t^{*}$ geometry. It is convenient to adopt the holomorphic gauge (2.19). We write $g_{a \bar{b}}$ for the $t t^{*}$ Hermitian metric on the fibers of the vacuum bundle $\mathscr{V}$ written in a holomorphic trivialization, and $\left(g_{q}\right)_{u \bar{v}}$ for its restriction to the sub-bundle $\mathscr{V}_{q} \subset \mathscr{V}$ of definite U(1) charge $q$, cf. (2.21), (2.25). For the sub-bundle $\mathscr{V}_{1-\hat{c} / 2} \cong T M$ we use the holomorphic local frame $\left\{\partial_{z^{j}}\right\}$ with $z^{j}$ complex coordinates on $M$; from now on indices from the middle of the latin alphabet $j, k, l, \ldots$ always refer to tensors defined in this holonomic holomorphic trivialization of $\mathscr{V}_{1-\hat{c} / 2} \subset \mathscr{V}$. Then, along the submanifold $M \hookrightarrow P$, one has [24, 30]

$$
K_{j \bar{k}}=\partial_{i} \bar{\partial}_{\bar{k}}\left(\sum_{q<0} 2 q \log \operatorname{det} g_{q}\right) .
$$

Eq. (2.32) says that the Hodge metric is the curvature of the Griffiths canonical bundle [21]

$$
\bigotimes_{q<0}\left(\operatorname{det} \mathscr{V}_{q}\right)^{-2 q} \rightarrow M \quad(\text { note that } 2 q \in \mathbb{Z})
$$

equipped with its canonical Chern connection [7]. For comparison, the WP metric is the curvature of the line bundle $\mathscr{V}_{-\hat{c} / 2} \rightarrow M[12,16]$

$$
G_{j \bar{k}}=-\partial_{j} \bar{\partial}_{\bar{k}} \log g_{-\hat{c} / 2} .
$$

The $t t^{*}$ equations yield a simple formula for the Riemann tensor of the WP metric on the conformal manifold of a $(2,2)$ SCFT. Taking the trace, we get a universal formula for the Ricci tensor valid on $M$ for all $\hat{c}$

$$
R_{j \bar{k}}=\left(C_{j} \bar{C}_{\bar{k}}\right)_{l}^{l}-(m+1) G_{j \bar{k}},
$$

where

$$
m \equiv \operatorname{dim}_{\mathbb{C}} M \equiv \operatorname{rank} \mathscr{V}_{1-\hat{c} / 2}
$$

In the special case $\hat{c}=3$ eq. (2.35) is sometimes called the 'Strominger formula' [12].

Writing $P_{q}$ for the orthogonal projection $\mathscr{V} \rightarrow \mathscr{V}_{q}$, we have

$$
\bar{\partial}_{\bar{k}} \partial_{j} \log g_{q}=-\operatorname{tr}\left(P_{q} \bar{\partial}_{\bar{k}}\left(g \partial_{j} g^{-1}\right)\right)=-\operatorname{tr}\left(P_{q}\left[C_{j}, \bar{C}_{\bar{k}}\right]\right),
$$

where in the last equality we used eq. (2.18) in the form ${ }^{16}$

$$
0 \equiv \operatorname{coeff} \zeta^{0} \text { in }\left[\left.\left(\nabla^{(\zeta)}\right)^{2}\right|_{(1,1) \text {-part }}\right]=D \bar{\partial}+\bar{\partial} D+C \wedge \bar{C}+\bar{C} \wedge C
$$

Setting $q=-\hat{c} / 2$ in (2.37) we recover the formula (2.34). The next case, $q=1-\hat{c} / 2$, yields

$$
\bar{\partial}_{\bar{k}} \partial_{j} \log g_{1-\hat{c} / 2}=G_{j \bar{k}}-\left(C_{j} \bar{C}_{\bar{k}}\right)_{l}^{l}=-R_{j \bar{k}}-m G_{j \bar{k}} .
$$

\footnotetext{
${ }^{16}$ Recall that $D \equiv d+\left(g \partial g^{-1}\right)$ is the $t t^{*}$ Chern connection [16].
} 
The same result may be obtained more directly by the first equation in (2.23)

$$
G_{j \bar{k}}=\frac{\left(g_{1-\hat{c} / 2}\right)_{j \bar{k}}}{g_{-\hat{c} / 2}} \Rightarrow \log \operatorname{det} G=\log \operatorname{det} g_{1-\hat{c} / 2}-m \log g_{-\hat{c} / 2},
$$

using the general Kähler identity $R_{j \bar{k}}=-\partial_{j} \bar{\partial}_{\bar{k}} \log \operatorname{det} G$, and eq. (2.34).

From eqs. (2.32), (2.34) and (2.39) we read the linear relations between the three tensors $G_{j \bar{k}}, K_{j \bar{k}}$, and $R_{j \bar{k}}$ on the moduli space of a Calabi-Yau $d$-fold. From the general discussion in section 2.1 we know that there are two linear relations for $d=1,2$ and one for $d=3,4$ :

$$
\begin{array}{lll}
d=1 & K_{j \bar{k}}=G_{j \bar{k}} & R_{j \bar{k}}=-2 G_{j \bar{k}} \\
d=2 & K_{j \bar{k}}=2 G_{j \bar{k}} & R_{j \bar{k}}=-m G_{j \bar{k}} \\
d=3 & K_{j \bar{k}}=(m+3) G_{j \bar{k}}+R_{j \bar{k}} & \\
d=4 & K_{j \bar{k}}=(2 m+4) G_{j \bar{k}}+2 R_{j \bar{k}} . &
\end{array}
$$

The first 3 lines are known to mathematicians [31] (eq. (2.43) was first derived in [24, 30]).

\subsection{Proof of eq. (1.10)}

By eq. (2.29) we may rewrite the linear relations between the tensors in arbitrary real local coordinates $x^{\alpha}$ since all three tensors have pure type $(1,1)$.

For $d=1$ eq. (2.41) implies (cf. (2.9))

$$
T_{\alpha \beta} \equiv K_{\alpha \beta}-\frac{1}{2} G_{\alpha \beta} G^{\gamma \delta} K_{\alpha \beta}=0
$$

which is the classical Virasoro constraint of the Polyakov world-sheet string action.

For $d=2$ the two equations (2.42) yield

$$
R_{\alpha \beta}-\frac{1}{2} G_{\alpha \beta} R-\kappa^{2} T_{\alpha \beta}=(m-1)\left(m+2 \kappa^{2}\right) G_{\alpha \beta}
$$

for all choice of $\kappa^{2}$.

For $d=3,4$ eqs. (2.43), (2.44) give

$$
T_{\alpha \beta} \equiv K_{\alpha \beta}-\frac{1}{2} G_{\alpha \beta} G^{\gamma \delta} K_{\gamma \delta}=\operatorname{gcd}(d, 2)\left(R_{\alpha \beta}-\frac{1}{2} G_{\alpha \beta} R\right)-(m-1)(\operatorname{gcd}(d, 2) m+d) G_{\alpha \beta} .
$$

Eqs. (2.45), (2.46), and (2.47) yield the various cases of (1.10).

\subsection{Finite actions and finite volume}

We have two distinct finite action statements. First, the $\sigma$-model action (2.27) is finite, that is, the moduli-space scalar field configuration, seen as a smooth map from $M$ to the target space $\Gamma \backslash G(\mathbb{R}) / K$, has finite energy in the sense of differential geometry. This holds for all CY dimension $d$. The second statement, valid for $d \leq 4$, is that the total gravity + matter action (1.1) is finite and proportional to the volume of the moduli $M$, so that the finite volume condition should be re-interpreted as a finite action requirement. 
The $\sigma$-model Lagrangian $L_{\sigma \text {-model }}$ evaluated on the particular solution describing the CY moduli geometry is just the WP trace of the Hodge metric (cf. eqs. (2.28), (2.30)), a quantity which is bounded by a constant outside a compact $U \Subset M$, as we see from the known asymptotics of VHS at infinity [21]. This shows the first statement.

For the relevant cases $d \leq 4$ we give an alternative and more direct argument. The cases $d=1,2$ are trivial, so we focus on $d=3$, 4. From eqs. (2.43), (2.44) we have

$$
\left.L_{\sigma \text {-model }}\right|_{t t^{*} \text { solution }} \equiv G^{\bar{k} j} K_{j \bar{k}}=\left(\frac{m}{\kappa^{2}}+d\right) m+\frac{1}{2 \kappa^{2}} R,
$$

where $m=\operatorname{dim}_{\mathbb{C}} M$ and $R$ is the moduli scalar curvature which is negative outside some compact $U$ [2]. Thus, outside the compact $U, 0 \leq L_{\sigma \text {-model }}<(m+3) m$ and

$$
\sigma \text {-model action }<\int_{U} d^{2 m} z \operatorname{det}\left(G_{j \bar{k}}\right) L_{\sigma \text {-model }}+\left(\frac{m}{\kappa^{2}}+d\right) m \cdot \operatorname{vol}(M \backslash U)<\infty,
$$

since the volume of $M$ is finite.

In the total action (1.1), the Einstein term $-R /\left(2 \kappa^{2}\right)$ cancels the last term in eq. (2.48) so that

$$
\text { total action }=\int_{M} d^{2 m} x \sqrt{G}\left(\frac{m}{\kappa^{2}}+d\right)=\left(\frac{m}{\kappa^{2}}+d\right) \cdot \operatorname{vol}(M),
$$

so that WP volume and total action agree up to overall normalization. In particular, since WP volumes of CY moduli spaces are finite [32], the total action is finite.

Remark 4. The total action is given by eq. (2.50) for all classical solutions of the model (1.1), not just for the particular one describing the moduli WP geometry.

\subsection{The canonical Kähler metrics for arbitrary $d$}

Consider the functions on $M$ of the form $(d \equiv \hat{c})$

$$
K^{(c)} \stackrel{\text { def }}{=} \sum_{q=-d / 2}^{d / 2} c(q) \log \operatorname{det} g_{q}, \quad c(q) \in \mathbb{R} .
$$

In a holomorphic gauge where $\operatorname{det} \eta=1$ (they exist [23]) the reality constraint [16] implies

$$
\log \operatorname{det} g_{q}=-\log \operatorname{det} g_{-q},
$$

so the function $(2.51)$ depends only on the $[(d+1) / 2]$ combinations $\{c(q)-c(-q)\}_{q<0}$, the same number as the dimension of the convex cone $\mathscr{C}_{d}$ of canonical metrics, and we are free to assume $c(q)$ to be an odd function. The Kähler potential of the WP metric has the form (2.51) with $c(q)= \pm 1 / 2$ for $q= \pm d / 2$ and zero otherwise. The Kähler potential of the Hodge metric has this form with $c(q)=q$, see eq. (2.32).

The general "God-given" Kähler metric in the cone $\mathscr{C}_{d}$ is then

$$
\begin{aligned}
K_{j \bar{k}}^{(c)} & \equiv \partial_{j} \partial_{\bar{k}}\left(\sum_{q=-d / 2}^{d / 2} c(q) \log \operatorname{det} g_{q}\right)=\operatorname{tr}\left[(c(Q+1)-c(Q)) C_{k} \bar{C}_{\bar{k}}\right] \equiv \\
& \equiv \sum_{q}(c(q+1)-c(q)) \operatorname{tr}\left(P_{q} C_{j} \bar{C}_{\bar{k}}\right) \equiv-\operatorname{tr}\left(\left[c(Q), C_{j}\right] \bar{C}_{\bar{k}}\right),
\end{aligned}
$$


where, as before, $P_{q}$ is the projector $\mathscr{V} \rightarrow \mathscr{V}_{q} . K_{j \bar{k}}^{(c)}$ is a positive Kähler metric when the coefficient function $c(q)$ belongs to the appropriate convex cone $\mathscr{C}_{d} \subset \mathbb{R}^{[(d+1) / 2]}$ which manifestly includes the cone of increasing functions $c(q+1)>c(q)$.

We show that all metrics $K_{j \bar{k}}^{(c)}$ satisfy the "Bianchi identity" (2.2). This property is automatic since these tensors correspond to $\partial \bar{\partial}$-exact $(1,1)$-forms $\kappa^{(c)} . \partial \kappa^{(c)}=0$ reads

$$
\begin{array}{ll}
D_{i} K_{j \bar{k}}^{(c)}=D_{j} K_{i \bar{k}}^{(c)} & D_{i}: \text { the }(1,0) \text { part of the } \\
& \text { WP Levi-Civita connection }
\end{array}
$$

Contraction with $G^{i \bar{k}}$ yields the "Bianchi identity".

\subsection{The explicit "Einstein equation" for arbitrary $d$}

From eq. (2.39) we get

$$
R_{j \bar{k}}-\frac{1}{2} G_{j \bar{k}} R-\left(1-m^{2}\right) G_{j \bar{k}}=\operatorname{tr}\left(P_{1-d / 2} C_{j} \bar{C}_{k}\right)-G_{j \bar{k}} G^{k \bar{l}} \operatorname{tr}\left(P_{1-d / 2} C_{k} \bar{C}_{l}\right)
$$

which is the explicit form of the linear relation (2.3). The tensor in the r.h.s. is conserved by the "Bianchi identity". While the r.h.s. looks as a valid energy-momentum tensor when evaluated on the on-shell configuration $S$, it is hard to find an off-shell action with positive kinetic terms which reproduces it. Our feeling is that it does not exist.

\section{Acknowledgments}

It is a pleasure to thank Cumrun Vafa for discussions, suggestions on the manuscript, and for a long time, fruitful collaboration which originated most of the ideas used in this note.

Open Access. This article is distributed under the terms of the Creative Commons Attribution License (CC-BY 4.0), which permits any use, distribution and reproduction in any medium, provided the original author(s) and source are credited.

\section{References}

[1] C. Vafa, The String landscape and the swampland, hep-th/0509212 [INSPIRE].

[2] H. Ooguri and C. Vafa, On the Geometry of the String Landscape and the Swampland, Nucl. Phys. B 766 (2007) 21 [hep-th/0605264] [INSPIRE].

[3] T.D. Brennan, F. Carta and C. Vafa, The String Landscape, the Swampland, and the Missing Corner, PoS TASI2017 (2017) 015 [arXiv:1711.00864] [INSPIRE].

[4] E. Palti, The Swampland: Introduction and Review, Fortsch. Phys. 67 (2019) 1900037 [arXiv: 1903.06239] [INSPIRE].

[5] P. Griffiths, Topics in Transcendental Algebraic Geometry, Princeton University Press (1984).

[6] P. Deligne, Travaux de Griffiths, Séminaire Bourbaki, vol. 1969/70 Exposés 364-381, Lecture Notes in Mathematics, vol. 180, Springer, Berlin, Heidelberg (1970) [DOI].

[7] J. Carlson, S. Müller-Stach and C. Peters, Period Mappings and Period Domains, Second Edition, Cambridge studies in advanced mathematics, vol. 168, Cambridge University Press (2017). 
[8] P. Griffiths, Mumford-Tate groups, notes available at https://publications.ias.edu/sites/default/files/Trieste.pdf.

[9] M. Green, P. Griffiths and M. Kerr, Mumford-Tate domains, Boll. Unione Mat. Ital. (Serie 9) 3 (2010) 281 [https://www.math.wustl.edu/ matkerr/MTD.pdf].

[10] M. Green, P. Griffiths and M. Kerr, Mumford-Tate Groups and Domains: Their Geometry and Arithmetic, Annals of Mathematics Studies, Princeton University Press (2012).

[11] S. Cecotti, $N=2$ Supergravity, Type IIB Superstrings and Algebraic Geometry, Commun. Math. Phys. 131 (1990) 517 [InSPIRE].

[12] A. Strominger, Special geometry, Commun. Math. Phys. 133 (1990) 163 [INSPIRE].

[13] S. Cecotti and C. Vafa, Theta-problem and the String Swampland, arXiv:1808.03483 [INSPIRE].

[14] S. Cecotti, Homogeneous Kähler Manifolds and T Algebras in $N=2$ Supergravity and Superstrings, Commun. Math. Phys. 124 (1989) 23 [InSPIRE].

[15] S. Cecotti, Special Geometry and the Swampland, JHEP 09 (2020) 147 [arXiv:2004.06929] [INSPIRE].

[16] S. Cecotti and C. Vafa, Topological antitopological fusion, Nucl. Phys. B 367 (1991) 359 [INSPIRE].

[17] S. Cecotti and C. Vafa, On classification of $N=2$ supersymmetric theories, Commun. Math. Phys. 158 (1993) 569 [hep-th/9211097] [INSPIRE].

[18] S. Cecotti, D. Gaiotto and C. Vafa, tt* geometry in 3 and 4 dimensions, JHEP 05 (2014) 055 [arXiv: 1312.1008] [INSPIRE].

[19] S. Cecotti, Geometry of $N=2$ Landau-Ginzburg families, Nucl. Phys. B 355 (1991) 755 [INSPIRE].

[20] C. Simpson, Higgs bundles and local systems, Publ. Math. IHES 75 (1992) 5.

[21] P.A. Griffiths, Periods of integrals on algebraic manifolds, III (Some global differential-geometric properties of the period mapping), Publ. Math. IHES 38 (1970) 125.

[22] W. Lerche, C. Vafa and N.P. Warner, Chiral Rings in $N=2$ Superconformal Theories, Nucl. Phys. B 324 (1989) 427 [InSPIRE].

[23] B. Dubrovin, Geometry of 2-D topological field theories, Lect. Notes Math. 1620 (1996) 120 [hep-th/9407018] [INSPIRE].

[24] S. Cecotti and C. Vafa, Ising model and $N=2$ supersymmetric theories, Commun. Math. Phys. 157 (1993) 139 [hep-th/9209085] [INSPIRE].

[25] K. Hori, A. Iqbal and C. Vafa, D-branes and mirror symmetry, hep-th/0005247 [INSPIRE].

[26] S. Cecotti, A. Neitzke and C. Vafa, Twistorial topological strings and a $\mathrm{tt}^{*}$ geometry for $\mathcal{N}=2$ theories in 4d, Adv. Theor. Math. Phys. 20 (2016) 193 [arXiv:1412.4793] [InSPIRE].

[27] J.A. Carlson and D. Toledo, Harmonic mappings of Kähler manifolds to locally symmetric spaces, Publ. Math. IHES 69 (1989) 173.

[28] B. Dubrovin, Geometry and integrability of topological-antitopological fusion, Commun. Math. Phys. 152 (1993) 539 [hep-th/9206037] [INSPIRE]. 
[29] S. Cecotti, Supersymmetric Field Theories. Geometric Structures and Dualities, Cambridge University Press (2015) [INSPIRE].

[30] M. Bershadsky, S. Cecotti, H. Ooguri and C. Vafa, Holomorphic anomalies in topological field theories, Nucl. Phys. B 405 (1993) 279 [hep-th/9302103] [INSPIRE].

[31] Z. Lu, On the Hodge metric of the universal deformation space of Calabi-Yau threefolds, math/0505582.

[32] Z. Lu and X. Sun, On the Weil-Petersson volume and the first Chern class of the moduli space of Calabi-Yau manifolds, Commun. Math. Phys. 261 (2006) 297 [math/0510021] [INSPIRE]. 